Pacific

Journal of

Mathematics

\title{
OBSTRUCTIONS TO DIAGONALIZATION MODULO LORENTZ IDEALS
}

H. Bercovici, L. Kérchy, AND R. Kovac 


\title{
OBSTRUCTIONS TO DIAGONALIZATION MODULO LORENTZ IDEALS
}

\author{
H. Bercovici, L. Kérchy, And R. Kovac
}

\begin{abstract}
We show that the results of David and Voiculescu (concerning the singular values of certain singular integral operators) yield obstruction results for a large class of ideals of compact operators. Diagonalization results which are counterparts of some of these obstructions were proved by Xia.
\end{abstract}

\section{Introduction.}

Let $T=\left(T_{1}, T_{2}, \ldots, T_{n}\right)$ be an $n$-tuple of commuting selfadjoint operators on a separable, complex Hilbert space $\mathfrak{H}$, and let $\mathfrak{J}$ be a separable symmetrically normed ideal of compact operators [2]. As usual, we say that $T$ is diagonalizable modulo $\mathfrak{J}$ if there exists an $n$-tuple $K=\left(K_{1}, K_{2}, \ldots, K_{n}\right)$ of operators in $\mathfrak{J}$ such that $T+K$ is diagonal in some orthonormal basis. Voiculescu introduced in [5] a systematic method for the treatment of diagonalizability. He proved that diagonalizability is equivalent with the existence of an approximate unit for the compact operators, which quasicommutes with $T$ modulo $\mathfrak{J}$. The obstruction to the existence of such a quasicentral approximate unit is given by the nonvanishing of a number $k_{\mathfrak{J}}(T) \in[0,+\infty]$. (We recall that $k_{\mathfrak{J}}(T)$ is the limit inferior of $\max \left\{\left|T_{j} A-A T_{j}\right|_{\mathfrak{J}}: 1 \leq j \leq n\right\}$ over the positive finite rank contractions $A$.) Thus $T$ is diagonalizable modulo $\mathfrak{J}$ if and only if $k_{\mathfrak{J}}(T)=0$. The number $k_{\mathfrak{J}}(T)$ is also defined for certain infinite commuting families of selfadjoint operators [7], and the equivalence between diagonalizability and $k_{\mathfrak{J}}=0$ was discussed for one such infinite family in [8].

Typically it is easier to calculate $k_{\mathfrak{J}}(T)$ when this number is zero. Results of the form $k_{\mathfrak{J}}(T) \neq 0$, also known as obstruction results, were obtained in [6], [1] by use of commutators. In fact Voiculescu showed in [7] that all obstruction results can in principle be obtained in this manner. More precisely, $k_{\mathfrak{J}}(T) \neq 0$ is equivalent to the existence of an $n$-tuple $X=\left(X_{1}, X_{2}, \ldots, X_{n}\right)$ from the dual ideal $\mathfrak{J}^{\prime}$ such that the commutator sum $\sum_{j=1}^{n}\left(T_{j} X_{j}-X_{j} T_{j}\right)$ is a trace-class perturbation of a positive operator and it has nonzero trace.

It is easy to see that diagonalizability of $T$ only depends on the scalar spectral measure of $T$. Therefore we can profitably restrict ourselves to the study of $n$-tuples which consist of multiplication by the $n$ variables in $L^{2}(\mu)$, where $\mu$ is a finite, positive, compactly supported Borel measure on $\mathbb{R}^{n}$. 
Denote by $X_{j}$ the integral operator with kernel $k(x, y)=\left(x_{j}-y_{j}\right) /|x-y|^{2}$. As already noted in [6], a formal calculation shows that $\sum_{j=1}^{n}\left(T_{j} X_{j}-X_{j} T_{j}\right)$ is an operator of rank one with nonzero trace. Thus an obstruction result can be obtained by showing that the operators $X_{j}$ belong to the ideal $\mathfrak{J}^{\prime}$. The operators $X_{j}$ often, though not always [1], provide the sharpest obstruction.

It was shown in [1] how to estimate the singular values of the operators $X_{j}$. The purpose of this note is to demonstrate how the results of [1] can help prove that $X_{j}$ belongs to a variety of Lorentz ideals, depending on the measure $\mu$. This will provide in particular obstructions which are the counterparts of certain diagonalization results in [8].

\section{Estimates of Singular Values.}

The estimate on the singular values of $X_{j}$ obtained in [1] are based on an abstract Hilbert space lemma, which we reproduce here in a somewhat modified version. The notation $|A|_{p}$ stands for the norm of the operator $A$ in the Schatten ideal $\mathfrak{S}_{p}$, and $s_{j}(A)$ denotes the $j$ th singular value of $A$ $\left(s_{j}(A)\right.$ is the distance from $A$ to the operators of rank $\left.<j\right)$.

Lemma 2.1 (Lemma 2.1 in [1]). Let $\left(\pi_{j}\right)_{j=1}^{\infty}$ be a decreasing sequence of positive numbers, $\left(m_{j}\right)_{j=1}^{\infty}$ an increasing sequence of positive integers, $\left(\beta_{j}\right)_{j=1}^{\infty}$ a sequence of positive numbers, $p \in[1, \infty)$, and $c \geq 1$. Assume that for $j=1,2, \ldots$ we have

(1) $m_{j+1} \leq c m_{j}$

(2) $\sum_{k=j+1}^{\infty} \beta_{k} \leq c \beta_{j}$

(3) $\sum_{k=1}^{j} \beta_{k} m_{k}^{1 / p} \leq c \beta_{j} m_{j}^{1 / p}$; and

(4) $\beta_{j} \leq c \pi_{m_{j}}$.

Assume further that $X$ is a compact operator which can be written as

$$
X=\sum_{j=1}^{\infty} \sum_{\ell=1}^{m_{j}} X_{j \ell}
$$

where $X_{j \ell} X_{j \ell^{\prime}}^{*}=X_{j \ell}^{*} X_{j \ell^{\prime}}=0$ for $\ell \neq \ell^{\prime}$, and $\left|X_{j \ell}\right|_{p} \leq \beta_{j}$ for $\ell=1,2, \ldots, m_{j}$. Then there exists a constant $C$, depending only on $c$ and $p$, such that

$$
s_{j}(X) \leq C \pi_{j}, \quad j \geq m_{1} .
$$

Proof. We provide the short argument because it requires a slight modification of the one given in [1]. Observe first that by (3) and (4)

$$
\beta_{j} m_{j}^{1 / p} \leq \sum_{k=1}^{j+1} \beta_{k} m_{k}^{1 / p} \leq c \beta_{j+1} m_{j+1}^{1 / p} \leq c^{2} \pi_{m_{j+1}} m_{j+1}^{1 / p} .
$$


This then yields

$$
\beta_{j} \leq c^{2}\left(\frac{m_{j+1}}{m_{j}}\right)^{1 / p} \pi_{m_{j+1}} \leq c^{2+1 / p} \pi_{m_{j+1}},
$$

a slight improvement of (4). If we set now $Y_{j}=\sum_{\ell=1}^{m_{j}} X_{j \ell}$ and $X_{j}=\sum_{i=1}^{j} Y_{i}$, we have

$$
\left|Y_{j}\right|_{p}=\left(\sum_{\ell=1}^{m_{j}}\left|X_{j \ell}\right|_{p}^{p}\right)^{1 / p} \leq\left(m_{j} \beta_{j}^{p}\right)^{1 / p}=m_{j}^{1 / p} \beta_{j},
$$

and

$$
\left\|Y_{j}\right\|=\max \left\{\left\|X_{j \ell}\right\|: 1 \leq \ell \leq m_{j}\right\} \leq \max \left\{\left|X_{j \ell}\right|_{p}: 1 \leq \ell \leq m_{j}\right\} \leq \beta_{j} .
$$

Observe now that

$$
\left|s_{i}(X)-s_{i}\left(X_{j}\right)\right| \leq\left\|X-X_{j}\right\| \leq \sum_{k=j+1}^{\infty}\left\|Y_{k}\right\| \leq \sum_{k=j+1}^{\infty} \beta_{k} \leq c \beta_{j},
$$

and

$$
\left|X_{j}\right|_{p} \leq \sum_{k=1}^{j}\left|Y_{k}\right|_{p} \leq \sum_{k=1}^{j} \beta_{k} m_{k}^{1 / p} \leq c \beta_{j} m_{j}^{1 / p},
$$

where we used inequalities (2) and (3). We have then

$$
\operatorname{card}\left\{i: s_{i}(X)>3 c \beta_{j}\right\} \leq \operatorname{card}\left\{i: s_{i}\left(X_{j}\right)>2 c \beta_{j}\right\} \leq\left[\frac{\left|X_{j}\right|_{p}}{2 c \beta_{j}}\right]^{p}<m_{j} .
$$

Thus, if $m_{j} \leq i \leq m_{j+1}$, we have

$$
s_{i}(X) \leq 3 c \beta_{j} \leq 3 c c^{2+1 / p} \pi_{m_{j+1}} \leq 3 c^{3+1 / p} \pi_{i} .
$$

The lemma follows with $C=3 c^{3+1 / p}$.

Assume now that $\mu$ is a positive, finite, compactly supported Borel measure on $\mathbb{R}^{n}$, and $h:[0,+\infty) \rightarrow[0,+\infty)$ is a continuous increasing function. The basic assumption on $\mu$ is that

$$
\mu(B) \leq h(r) \text { if } B \text { is a ball of radius } r .
$$

The function $h$ will be subject to conditions

$$
\begin{gathered}
h(2 r) \leq \operatorname{ch}(r) ; \\
\frac{h(r)}{r} \downarrow 0 \text { as } r \downarrow 0 ; \quad \text { and } \\
\sum_{k=n+1}^{\infty} 2^{k} h\left(2^{-k}\right) \leq c 2^{n} h\left(2^{-n}\right), \quad n=1,2, \ldots
\end{gathered}
$$

for some constant $c>0$. Since $\mu$ is compactly supported, the function $h$ will be assumed eventually constant. Note that (2.4) and (2.5) are verified 
for small $r$ if, for instance, $h(t)=t^{1+\varepsilon} g(t)$, where $\varepsilon>0$ and $g$ is increasing near the origin. Observe that condition (2.4) implies that the sequence

$$
\frac{1}{n h^{-1}\left(\frac{1}{n}\right)}, \quad n=1,2, \ldots
$$

is decreasing. In view of this fact and of the improved form of Lemma 2.1, Theorem 3.1 of [1] can be restated as follows. The operators $X_{j}$ are the integral operators described in Section 1.

Theorem 2.6. Given $\mu$ and $h$ as above, there exists a constant $C>0$ such that

$$
s_{k}\left(X_{j}\right) \leq \frac{C}{k h^{-1}\left(\frac{1}{k}\right)}, \quad k=1,2, \ldots, j=1,2, \ldots, n .
$$

\section{Obstructions for Lorentz Ideals.}

Let us recall that, given $p, q \in(0,+\infty)$, the collection $\mathfrak{S}_{p, q}$ of all compact operators $K$ satisfying

$$
\sum_{j=1}^{\infty} j^{-1+q / p} s_{j}(K)^{q}<\infty
$$

is an ideal. The ideal $\mathfrak{S}_{p, q}$ is symetrically normed if $p, q \geq 1$ ([4]; see also $[3])$.

Our main result establishes sufficient conditions for the operators $X_{j}$ described in the introduction to belong to $\mathfrak{S}_{p, q}$. The measure $\mu$ and the function $h$ will be as in Section 2 .

Theorem 3.1. Assume that $p, q \geq 1$ and

$$
\int_{0}^{1} \frac{h(r)^{q(1-1 / p)}}{r^{q+1}} d r<\infty .
$$

Then the operators $X_{j}$ belong to $\mathfrak{S}_{p, q}$.

Proof. Assume the integral in the statement is finite. By Theorem 2.6, it suffices to show that the sum

$$
\sum_{j=1}^{\infty} j^{-1+q / p}\left(\frac{1}{j h^{-1}\left(\frac{1}{j}\right)}\right)^{q}=\sum_{j=1}^{\infty} \frac{j^{-1-q+q / p}}{\left(h^{-1}\left(\frac{1}{j}\right)\right)^{q}}
$$

is finite. Since the function $x^{-1-q+q / p}$ does not vary too much on intervals of the form $(j, j+1)$, the above sum is finite if and only if the integral

$$
\int_{1}^{\infty} \frac{x^{-1-q+q / p}}{\left(h^{-1}\left(\frac{1}{x}\right)\right)^{q}} d x=\int_{0}^{1} \frac{y^{-1+q-q / p}}{h^{-1}(y)^{q}} d y
$$


is finite. The substitution $h^{-1}(y)=r$ shows that this integral is finite if and only if

$$
\int_{0}^{1} \frac{h(r)^{-1+q-q / p}}{r^{q}} d h(r)
$$

is finite. This integral is (up to a constant)

$$
\int_{0}^{1} \frac{d h(r)^{q-q / p}}{r^{q}}
$$

and this is finite if and only if

$$
\int_{0}^{1} \frac{h(r)^{q(1-1 / p)}}{r^{q+1}} d r<\infty .
$$

The theorem follows.

Let us look at two particular cases. First, if $p=q>1$ then $\mathfrak{S}_{p, p}$ is the Schatten ideal $\mathfrak{S}_{p}$. The condition in Theorem 3.1 is that

$$
\int_{0}^{1} \frac{h(r)^{p-1}}{r^{p+1}} d r<\infty
$$

This is therefore an obstruction to diagonalization modulo the ideal $\mathfrak{S}_{p^{\prime}}$, where $p^{\prime}=p /(p-1)$ is the conjugate exponent. If the integral is infinite and inequality (2.2) is reversed, diagonalization is in fact possible (cf. Theorem 8.1 in $[8]$ ). In subsequent work Xia [9] studied in more detail the decomposition of the operators $X_{j}$ given in [1], and thereby obtained necessary and sufficient conditions for diagonalization modulo $\mathfrak{S}_{p^{\prime}}$ in terms of $\mu$ alone.

Next consider the case $q=1, p>1$. The condition in Theorem 3.1 becomes

$$
\int_{0}^{1} \frac{h(r)^{1-1 / p}}{r^{2}} d r<\infty
$$

Again, this is an obstruction result for diagonalization modulo a certain ideal $\mathfrak{S}_{p^{\prime}, \infty}^{(0)}$ whose dual is $\mathfrak{S}_{p, 1}$. (We recall that a compact operator $X$ belongs to $\mathfrak{S}_{p^{\prime}, \infty}^{(0)}$ if and only if $\lim _{j \rightarrow \infty} j^{1 / p^{\prime}} s_{j}(X)=0$.) If the integral is infinite then the diagonalization result is Theorem 8.4 in [8].

These examples suggest that there is good reason to believe that the obstruction results given by Theorem 3.1 are sharp, in the sense that diagonalization is always possible when the corresponding integral is infinite and (2.2) is reversed. 


\section{References}

[1] G. David and D. Voiculescu, s-numbers of singular integrals for the invariance of absolutely continuous spectra in fractional dimensions, J. Funct. Anal., 94 (1990), 14-26.

[2] I. Gohberg and M. Kreŭn, Introduction to the Theory of Linear Nonselfadjoint Operators, Translations of Mathematical Monographs, 18, Amer. Math. Soc., Providence, Rhode Island, 1969.

[3] R. Kovac, Composition Operators in Lorentz Ideals, Thesis, Indiana University, 1996.

[4] B. Simon, Trace Ideals and their Applications, London Math. Soc. Lecture Notes, No. 35, Cambridge Univ. Press, Cambridge, 1979.

[5] D. Voiculescu, Some results on norm-ideal perturbations of Hilbert space operators, J. Operator Theory, 2 (1979), 3-37.

[6] _ Some results on norm-ideal perturbations of Hilbert space operators, II, J. Operator Theory, 5 (1981), 77-100.

[7] _ On the existence of quasicentral approximate units relative to norm ideals, I, J. Funct. Anal., 91 (1990), 1-36.

[8] J. Xia, Diagonalization modulo norm ideals with Lipschitz estimates, J. Funct. Anal., 145 (1997), 491-526.

[9] _ Diagonalization and unitary equivalence modulo Schatten p-classes, preprint.

Received March 31, 1998. The first named author was supported in part by a grant from the National Science Foundation. The second named author was visiting Indiana University when work on this paper was completed.

INDIANA UNIVERSITY

BloOmington, IN 47405

E-mail address: bercovic@indiana.edu

Bolyai Institute, University of Szeged

6720 SZEGED

HUNGARY

E-mail address: kerchy@math.u-szeged.hu

University of Minnesota-Duluth

Duluth, MN 55812

E-mail address: rkovac@d.umn.edu 